\title{
COMPARISON OF ECHOCARDIOGRAPHIC FEATURES OF PATIENTS WITH INFECTIVE ENDOCARDITIS IN HIV-POSITIVE TUBERCULOSIS PATIENTS AND HIV-NEGATIVE TUBERCULOSIS PATIENTS
}

\author{
P. V. Kalyan Kumar', Ramakrishna Gorantla², Ramakrishna Rachakonda ${ }^{3}$ \\ ${ }^{1}$ Assistant Professor, Department of Respiratory Medicine, Katuri Medical College, Guntur, Andhra Pradesh. \\ ${ }_{2}^{2}$ Associate Professor, Department of General Medicine, Katuri Medical College, Guntur, Andhra Pradesh. \\ 3 Professor, Department of Pulmonary Medicine, Katuri Medical College, Guntur, Andhra Pradesh.
}

\section{ABSTRACT}

\section{BACKGROUND}

Infective endocarditis (IE) is an infection of the endocardial surface of the heart structures, and is caused by the microbial bacterial organisms and fungal infection that occurs as a result of colonisation of the endothelium by microorganisms. The following study intended to evaluate the echocardiographic features of infective endocarditis on immunocompromised patients.

The aim of this study is to compare the echocardiographic features of patients with infective endocarditis in HIV-positive tuberculosis patients and HIV-negative tuberculosis patients.

\section{MATERIALS AND METHODS}

This was a retrospective descriptive observational study, conducted over two years from April 2015-April 2017 of all the infective endocarditis patients using the modified Duke criteria for diagnosing IE. Patients having PTB and HIV are included in the study. All tuberculosis patients are divided into two groups based on the HIV status.

\section{RESULTS}

During this period of two years, 91 patients were screened for infective endocarditis. 77 satisfied the criteria for a definite diagnosis of IE. Most patients had advanced valve disruption with heart failure. 38 patients were diagnosed as pulmonary tuberculosis or extrapulmonary based on molecular testing. Of them, 26 patients were HIV-positive tuberculosis patients, and 8 were HIV-negative tuberculosis patients. Most of the clinical features in the two groups of patients were similar. Vegetations were the predominant finding in 34 patients on echocardiography. Vegetations were found in 26 of the HIV-positive pulmonary tuberculosis patients, and in 8 of the HIV-negative tuberculosis patients. This clearly shows HIV and TB co-infection leads to significant increased incidence of infective endocarditis when compared to tuberculosis itself. The leaflet aneurysms appeared larger in size in the HIV-positive tuberculosis patients $(0.92 \mathrm{~cm}$ depth $\mathrm{x} 0.91 \mathrm{~cm}$ width) when compared to the smaller-sized aneurysm in the HIV-negative patients $(0.14 \mathrm{~cm}$ depth $\mathrm{x} 0.6 \mathrm{~cm}$ width) which is statistically significant.

\section{CONCLUSION}

There was no difference in the clinical presentation of infective endocarditis between HIV-positive and HIV-negative tuberculosis patients. Vegetations were more common in HIV-positive cohort when compared to HIV-negative cohort. Leaflet aneurysms were also more common in the HIV-positive tuberculosis patients when compared to HIV-negative tuberculosis patients.

\section{KEYWORDS}

Human Immunodeficiency Virus (HIV), Pulmonary Tuberculosis (PTB), Infective Endocarditis (IE), Duke Criteria, Vegetations, Extrapulmonary TB (EPTB).

HOW TO CITE THIS ARTICLE: Kumar PVK, Gorantla R, Rachakonda R. Comparison of echocardiographic features of patients with infective endocarditis in HIV-positive tuberculosis patients and HIV-negative tuberculosis patients. J. Evolution Med. Dent. Sci. 2017;6(78):5536-5539, DOI: 10.14260/jemds/2017/1203

\section{BACKGROUND}

Infective endocarditis (IE) is an infection of the endocardial surface of the heart structures, and is caused by the microbial bacterial organisms and fungal infection that occurs as a result of colonisation of the endothelium by microorganisms. Major predisposing factors to endocarditis are underlying acquired or congenital cardiac valvular abnormalities, such as

'Financial or Other Competing Interest': None.

Submission 23-08-2017, Peer Review 16-09-2017,

Acceptance 23-09-2017, Published 28-09-2017.

Corresponding Author:

Dr. P. V. Kalyan Kumar

Assistant Professor,

Department of Pulmonary Medicine,

Katuri Medical College and Hospital,

Guntur,

Andhra Pradesh.

E-mail: drpvkalyan@hotmail.com

DOI: $10.14260 /$ jemds/2017/1203

acquired or congenital cardiac valvular abnormalities, such as rheumatic heart disease, including the presence of prosthetic valves in heart, and central venous access catheters which provide a nidus for the infectious organism. IE is characterised by many clinical features, none of which are specific for the condition, and therefore the diagnosis is determined by the presence of multiple findings. Important developments during the last 2 decades have facilitated rapid and accurate diagnosis, and have shown that early aggressive treatment is associated with improved survival in most of the patients.

Major trends that have created new challenges are emergence of prosthetic valve endocarditis, intravascular device-related endocarditis, an increase in antibiotic resistance among causative organisms.

Patients with comorbid conditions such as diabetes, dialysis-dependent renal failure, and intravenous drug abuse and the immune compromised status associated with acquired immune deficiency syndrome and pulmonary 
tuberculosis masquerade diagnostic challenges that also have important implications for treatment of IE Patients with poor dental hygiene and diabetes mellitus are also conditions associated with an increased incidence of infective endocarditis, mainly the profound immunosuppression found in AIDS along with pulmonary tuberculosis predisposes these patients to multiple opportunistic infections including IE.

\section{MATERIALS AND METHODS}

The study was a retrospective observational cohort study in which a total of 91 patients with features of suspected IE were included in the study over two years from April 2015April 2017 using the modified Duke criteria for diagnosing of IE. Patients having PTB and HIV are included in the study. All tuberculosis patients are divided in to two groups based on the HIV status.

Only patients with a definite diagnosis of IE were included in the study.

Patients with a clinical diagnosis of IE had undergone transthoracic echocardiography (TTE) to diagnose echocardiographic evidence of infection namely vegetations, paravalvular extension, prosthetic valve dehiscence, check haemodynamic compromise based on valvular dysfunction, ventricular dimensions and ventricular ejection fraction.

When TTE images were suboptimal as in case of mechanical prosthetic valves, and if paravalvular extension was suspected, trans-oesophageal echocardiography (TEE) was performed.

The diagnosis of IE was made by clinical criteria including echocardiography and based on pathological results.

\section{Clinical Criteria}

The clinical criteria (modified Duke criteria) consists of major and minor criteria for definitive diagnosis- 2 major or 1 major and 2 minor or 5 minor criteria.

\section{These Criteria are as Follows}

Major Criteria-

1. Positive blood cultures with organisms typical for endocarditis.

2. Evidence of endocardial involvement.

\section{Minor Criteria-}

1. Predisposing heart disease.

2. Fever $>38^{\circ} \mathrm{C}$.

3. Vascular and immunological phenomena.

4. Microbiological evidence not meeting major criteria.

5. Elevated erythrocyte sedimentation rate (ESR) and C-reactive protein (CRP) levels.

6. Splenomegaly, clubbing, splinter haemorrhages, and petechiae.

7. Central and peripheral venous access.

8. Organisms from metastatic lesions and haematuria.

\section{Statistical Analysis}

Data analysis was done by SPSS 24.0. Chi-squared test was used for categorical data and to find the statistical significance of the two main variables namely the vegetations and Leaflet aneurysms.

\section{RESULTS}

During this period of two years, 91 patients were screened for infective endocarditis. 77 satisfied the criteria for a definite diagnosis of IE. Most patients had advanced valve disruption with heart failure. 38 patients were diagnosed as pulmonary tuberculosis or extrapulmonary based on molecular testing. Of them, 26 patients were HIV-positive tuberculosis patients, and 8 were HIV-negative tuberculosis patients.

Both pulmonary and extrapulmonary tuberculosis patients were added. Pulmonary TB was based on sputum positivity or molecular diagnosis namely TB NAAT or clinical suspicion. Extrapulmonary TB was based on tissue biopsy. Blood culture for TB was not done in our study.

Most of the patients' chest X-rays are mostly atypical with lobar infiltrates in the midzone and lower zone followed by cavitations in the upper zone.

All patients $(n=38)$ had findings suggestive of infective endocarditis on TTE. These positive findings include the presence of vegetations, root abscesses or leaflet aneurysms. Overall, 15 patients had echocardiographic features suggestive of rheumatic heart disease. Of these, 10 were HIV negative and 5 were HIV positive. These echocardiographic features included thickened valves, failure of leaflet coaptation and leaflet prolapse, which were regarded as possible predisposing factors to infection. Congenital defects were found in 4 patients, two of whom were patients with ASD ( 1 HIV positive and 1 HIV negative). Of the remaining 2 patients, 1 had a bicuspid aortic valve, and the other a PDA, both of whom were HIV negative.

\section{Vegetations}

Vegetations were the predominant finding in 34 patients on echocardiography. Vegetations were found 26 of the HIVpositive pulmonary tuberculosis patients, and in 8 of the HIVnegative pulmonary tuberculosis patients. The remaining 4 patients (2 HIV-positive and 2 HIV-negative patients) showed no evidence of any vegetation, but had other features suggestive of IE. These were leaflet aneurysms in 2 patients and aortic root abscesses in remaining 2 patients.

\section{Leaflet Aneurysms}

Leaflet aneurysms were found in four of the 20 HIV-positive patients, affecting both the mitral and aortic valves with predominantly aortic valve. Among the 18 HIV-negative patients, one had a leaflet aneurysm associated with vegetations, affecting the mitral valve only. The leaflet aneurysms appeared larger in size in the HIV-positive patients $(0.92 \mathrm{~cm}$ depth $\times 0.91 \mathrm{~cm}$ width), compared to the smaller-sized aneurysm in the HIV-negative patient $(0.14 \mathrm{~cm}$ depth $\mathrm{x} 0.6 \mathrm{~cm}$ width) $(\mathrm{p}=.006)$.

\begin{tabular}{|c|c|c|c|c|}
\hline Features & $\begin{array}{c}\text { HIV + \& } \\
\text { PTB }\end{array}$ & $\begin{array}{c}\text { HIV - \& } \\
\text { PTB }\end{array}$ & $\begin{array}{c}\text { Total } \\
\text { Patients }\end{array}$ & P value \\
\hline Vegatations & $26(76 \%)$ & $08(24 \%)$ & 34 & $\mathrm{P}<0.001$ \\
\hline $\begin{array}{c}\text { Valve } \\
\text { Aneurysm }\end{array}$ & $04(80 \%)$ & $01(20 \%)$ & 05 & $\mathrm{P}<0.006$ \\
\hline
\end{tabular}

Table 1. Echocardiographic Features in both the Groups

\section{Other Complications}

Extensive valve disruption was present in both cohorts, since patients presented at a very advanced stage of co-infection. 
Except for leaflet aneurysms and vegetations, which were present in four and 26 HIV-positive co-infected patients respectively, there did not appear to be any difference in the prevalence of valve-related complications like valve disruption due to chordal rupture, and perforated or flail leaflets between the two groups and were equally distributed in the two groups. There were striking differences with regards to the shape of vegetations where HIV-positive coinfected PTB have large vegetation size when compared to the other group. In both groups, ejection fraction was good. Clinical heart failure with fluid overload was same in both the groups. Chi-squared test used to compare the vegetations, and leaflet aneurysms show significant difference between the two groups $(\mathrm{p}<0.05)$.

\section{DISCUSSION}

According to Levy et al, the risk of Human Immunodeficiency Virus (HIV) positive patients with pulmonary tuberculosis developing infective endocarditis is related to their degree of immunodeficiency. ${ }^{1}$ The decreased CD4 common to HIV patients is likely to increase their risk of developing endocarditis. Very little else is known about the nature of the infecting organisms and the pattern of disease in the immune-suppressed HIV-positive pulmonary tuberculosis patients. $^{2}$ Neither is much known about the nature of complicating IE in patients with pulmonary tuberculosis without underlying HIV. ${ }^{3}$

A precise diagnosis of IE is mandatory to guide therapy. Rapid diagnosis, effective treatment and prompt recognition of complications are essential for a good outcome of infective endocarditis, which carries a high morbidity and mortality rate. ${ }^{4}$

Mycobacterium tuberculosis (TB) is a major cause of death worldwide. Tuberculosis is capable of infecting any organ of the body, and even the heart is no exception. ${ }^{5}$ Tuberculous endocarditis (TBE) was first reported in $19^{\text {th }}$ century and subsequently many other case series have been described, highlighting the significant morbidity and mortality associated with this manifestation of tuberculosis. TBE usually presents with miliary disseminated tuberculosis. ${ }^{6}$ With increasing application of prosthetic valve replacements in the interventional cardiology, TB infections have begun to affect these as well as native valves. ${ }^{7,8}$ With the introduction of diagnosing TB by molecular methods and effective drug therapy, the prognosis has improved. HIV and drug resistance are likely to make the management of TBE.

There have been few studies that have prospectively examined IE in HIV and TB co-infected subjects, where infective endocarditis is more common. In contrast to the Western series, we did not identify intravenous drug abuse as a risk factor but rheumatic heart disease remains as an important predisposing factor for developing endocarditis in 15 patients. In developing countries like India where rheumatic heart disease is endemic and repeated infection is common leading to IE. Considerable skill is needed in differentiating vegetations from damage due to previous infection disease or rheumatic carditis, and in differentiating vegetations from damaged chordal apparatus which is frequent in patients with valvular heart disease.

Furthermore, patients with HIV infection already have elevated ESR and CRP and concurrent anaemia from antecedent infection rendering diagnostic difficulties in evaluating IE.

Vegetations were the predominant finding in 34 patients on echocardiography. Vegetations were found in 26 of the HIV-positive pulmonary tuberculosis patients, and in 8 of the HIV-negative pulmonary tuberculosis patients. This clearly shows HIV and TB co-infection leads to significant increased incidence of infective endocarditis when compared to tuberculosis itself.

The leaflet aneurysms appeared larger in size in the HIV tuberculosis-positive patients $(0.92 \mathrm{~cm}$ depth $\times 0.91 \mathrm{~cm}$ width) when compared to the smaller-sized aneurysm in the HIV-negative patient $(0.14 \mathrm{~cm}$ depth $\times 0.6 \mathrm{~cm}$ width $)$ which is statistically significant.

\section{CONCLUSION}

This is one of the first echocardiographic studies of IE comparing HIV-positive pulmonary tuberculosis patients and HIV-negative PTB patients in a cohort of 38 patients diagnosed IE based on the modified Duke criteria. Few data have been published on the clinical characteristics and outcome of IE in patients with HIV+PTB infection. Even fewer data exist on the echocardiographic findings in these patients. To date, IE in HIV+PTB infected individuals in Western series has been described almost exclusively in intravenous drug users, and has been reportedly rare in other HIV+PTB infected subjects. In this report, we have described the echocardiographic features of IE in HIV+PTB positive subjects and related them to the HIV-negative PTB patients.

In contrast to Western series, the most common underlying predisposing abnormality observed in our study was rheumatic heart disease. The mitral and aortic valves were predominantly affected, mostly by the presence of vegetations. Certain differences emerged in that the size of the vegetations were slightly larger in the HIV-positive 0.92 $\mathrm{cm}$ depth $\mathrm{x} 0.91 \mathrm{~cm}$ width) when compared to the smallersized aneurysm in the HIV-negative patients $(0.14 \mathrm{~cm}$ depth $\mathrm{x}$ $0.6 \mathrm{~cm}$ width) which is statistically significant, a finding that was more predominant in the patients classified as having AIDS. Leaflet aneurysms and aortic root abscesses occurred more frequently in the HIV-positive patients, when compared to HIV-negative TB patients.

With the exception of fever which was significantly more common the HIV-positive patients (23.5\%), the clinical profile of IE in the HIV-positive patients was similar to that of HIV-negative patients, and was characterised by clubbing, murmurs and severe valve regurgitation. Echocardiography revealed severe valve damage, often with abscess formation, consequent upon advanced infection. Although ventricular contractility, as assessed by EF was similar in both groups.

\section{REFERENCES}

[1] Levy WS, Simon GL, Rios JC, et al. Prevalence of cardiac abnormalities in human immunodeficiency virus infection. Am J Cardiol 1989;63(1):86-9.

[2] Sultan FA, Fatimi S, Jamil B, et al. Tuberculous endocarditis: valvular and right atrial involvement. Eur J Echocardiogr 2010;11(4):E13.

[3] Alkhuja S, Miller A. Tuberculosis and sudden death: a case report and review. Heart Lung 2001;30(5):38891. 


\section{Jemds.com}

[4] Hoen B, Duval X. Infective endocarditis. N Engl J Med 2013;369(8):785.

[5] WHO global tuberculosis control report 2010. Summary. Cent Eur J Public Health 2010;18(4):237.

[6] Barrett AM, Cole L. A case of tuberculous pericarditis. Br Heart J 1944;6(4):185-90.
Original Research Article

[7] Yamane H, Fujiwara T, Doko S, et al. Two cases of miliary tuberculosis following prosthetic valve replacement. Kokyu To Junkan 1989;37(7):803-5.

[8] Warwick RM, Magee JG, Leeming JP, et al. Mycobacteria and allograft heart valve banking: an international survey. J Hosp Infect 2008;68(3):255-61. 